\title{
Regulation of tumor angiogenesis by the microtubule-binding protein CLIP-170
}

\author{
Xiaodong Sun, Fang Li, Bin Dong, Shaojun Suo, Min Liu, Dengwen Li, Jun Zhou ${ }^{凶}$ \\ Department of Genetics and Cell Biology, College of Life Sciences, Nankai University, Tianjin 300071, China \\ $\triangle$ Correspondence: junzhou@nankai.edu.cn. \\ Received January 20, 2013 Accepted March 1, 2013
}

\begin{abstract}
Angiogenesis, the expansion of preexisting blood vessels, is a complex process required for tumor growth and metastasis. Although current antiangiogenic strategies have shown promising results in several cancer types, identification of additional antiangiogenic targets is required to improve the therapeutic response. Herein, we show that the microtubule-binding protein CLIP-170 (cytoplasmic linker protein of $170 \mathrm{kDa}$ ) is highly expressed in breast tumor samples and correlates positively with blood vessel density. Depletion of CLIP-170 significantly impaired vascular endothelial tube formation and sprouting in vitro and inhibited breast tumor growth in mice by decreasing tumor vascularization. Our data further show that CLIP170 is important for the migration but not the proliferation of vascular endothelial cells. In addition, CLIP-170 promotes the polarization of endothelial cells in response to the angiogenic stimulus. These findings thus demonstrate a critical role for CLIP-170 in tumor angiogenesis and suggest its potential as a novel antiangiogenic target.
\end{abstract}

KEYWORDS tumor angiogenesis, endothelial cell, migration, polarization, microtubule

\section{INTRODUCTION}

Angiogenesis refers to a multistep process of neovasculature formation from the preexisting vascular network and is essential for blood vessel remodeling during development and wound healing (Carmeliet and Jain, 2011). In addition, a wealth of compelling evidence has demonstrated a critical requirement for angiogenesis in both progressive growth and metastasis of tumors (Folkman, 2007; Carmeliet and Jain, 2011; Weis and Cheresh, 2011). The angiogenic process is controlled by proangiogenic and antiangiogenic factors in both physiological and pathological situations. Tumor-associated angiogenesis is orchestrated by the tumor microenvironment, in which tumor cells can shift the balance toward proangiogenic factors to stimulate the sprouting of blood vessels. Specifically, the angiogenic switch is triggered by the release from tumor cells of various growth factors, cytokines, and chemokines, which activate the surrounding cells to initiate neovascularization (Folkman, 2007; Weis and Cheresh, 2011).

Endothelial cells represent one of the major cell types surrounding tumor cells. In response to angiogenic signals, endothelial cells in the nearby blood vessels migrate and proliferate to form provisional tubes (Lamalice et al., 2007). This is followed by the recruitment and activation of perivascular cells, which cover the endothelial tubes to form stable and mature blood vessels (Lamalice et al., 2007; Carmeliet and Jain, 2011). The migration of vascular endothelial cells thus is a key step in the angiogenic process and is required for vascular sprouting. Research in the past decade has provided important insights into the pattern and regulation of cell migration (Gao et al., 2010; Vicente-Manzanares and Horwitz, 2011; Li et al., 2012; Shi et al., 2012). In the tumor microenvironment, the gradients of proangiogenic factors can induce the polarization of vascular endothelial cells, with the appearance of protrusions at the cell front, asymmetric localization of signaling molecules, and reorganization of cellular structures. The polarization of vascular endothelial cells is important for their efficient migration toward tumor cells (Vicente-Manzanares and Horwitz, 2011).

There is accumulating evidence that cytoskeletal components, mainly actin filaments and microtubules, are critical for cell polarization and migration (Rodriguez et al., 2003; Watanabe et al., 2005; Li and Gundersen, 2008). While actin filaments act as the physical backbone of protrusion and provide the main force of migration, microtubules define the direction of polarization in concert with the nucleus (Rodriguez et al., 2003; Watanabe et al., 2005; Li and Gundersen, 2008; Shi et 
A

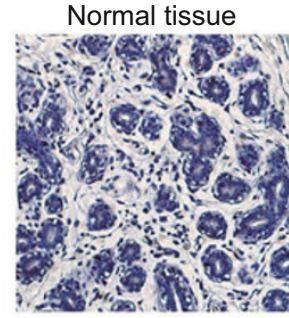

B

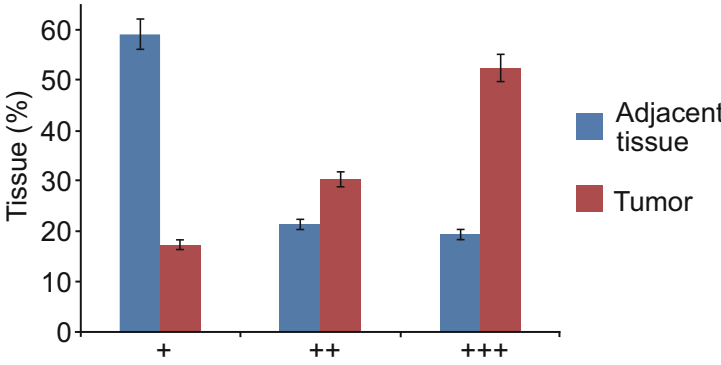

D
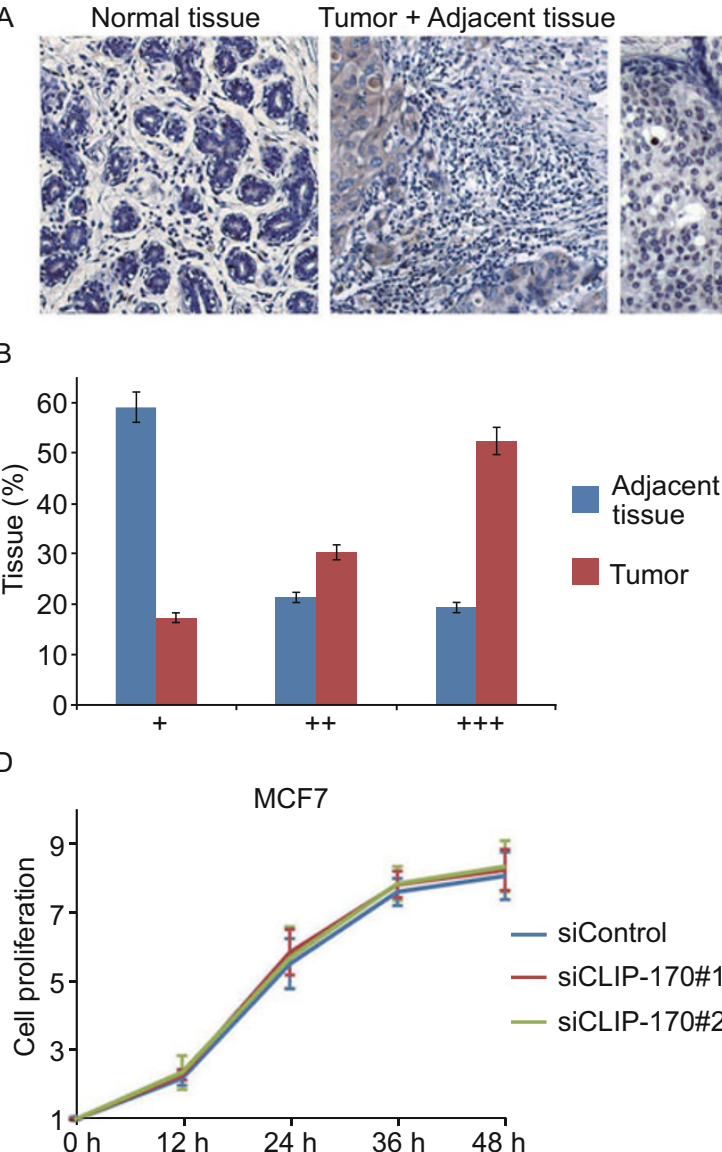

\section{$+$}

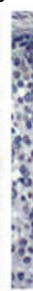

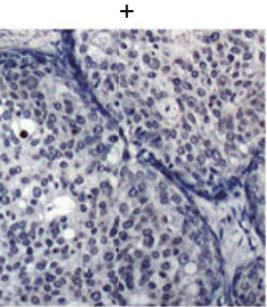

C

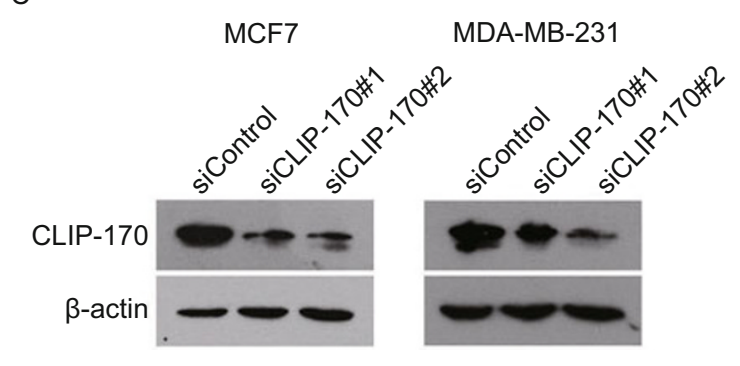

E

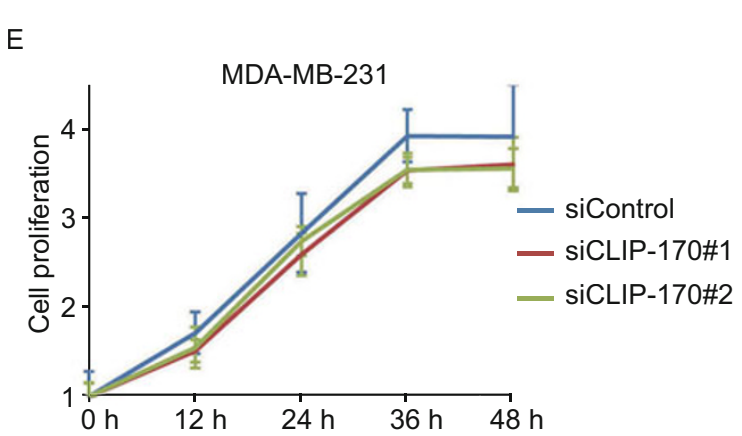

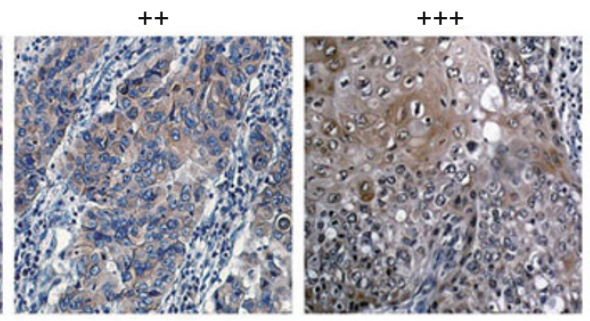

MDA-MB-231

Figure 1. CLIP-170 is highly expressed in human breast carcinoma but is not critically involved in cancer cell proliferation. (A) Immunohistochemistry of CLIP-170 expression in normal breast tissue, breast carcinoma, and adjacent tissue samples. Representative images of breast carcinoma samples with low (+), medium (++), and high (+++) expression of CLIP-170 are shown. (B) Quantification of breast carcinoma and adjacent tissue samples with low, medium, or high expression of CLIP-170. (C) Western blot analysis of CLIP170 and $\beta$-actin expression in MCF7 and MDA-MB-231 cells transfected with CLIP-170 or control siRNAs for $72 \mathrm{~h}$. (D) MCF7 and (E) MDA-MB-231 cells were transfected with CLIP-170 or control siRNAs and cell proliferation was examined by sulforhodamine $B$ staining.

al., 2012). In migrating cells, microtubule plus ends radiate primarily toward the cell front and minus ends are concentrated at the centrosome, which is positioned between the nucleus and the cell front (Bornens, 2008). This specific organization of microtubules is achieved via the capture and stabilization of microtubule plus ends by the cell cortex and mediated by microtubule plus end-tracking proteins collectively named +TIPs (Akhmanova and Steinmetz, 2008; Gouveia and Akhmanova, 2010; Li et al., 2011; Peng et al., 2012). CLIP-170 is a founding member of the +TIPs family and participates in cell migration by mediating the cortical capture of microtubules (Gouveia and Akhmanova, 2010). CLIP-170 has also been implicated in the pathogenesis of cancer; however, the underlying molecular mechanisms remain obscure (Bilbe et al., 1992; Delabie et al., 1992; Suzuki and Takahashi, 2008; Sun et al., 2012). In this study, our data demonstrate that CLIP-170 stimulates tumor angiogenesis by mediating vascular endothelial cell polarization and migration, and establish a critical link between the pathological role of CLIP-170 and its role as a component of the microtubule-cell migration axis.

\section{RESULTS}

CLIP-170 is highly expressed in human breast carcinoma but is not critically involved in cancer cell proliferation

To examine the potential involvement of CLIP-170 in the pathogenesis of breast cancer, we analyzed its expression in normal breast tissue, breast carcinoma, and adjacent tissue by immunohistochemistry. CLIP-170 was expressed at a low level in all the normal breast tissue and most adjacent tissue samples examined (Fig. 1A and 1B). In contrast, a significant increase in CLIP-170 expression was observed in breast carcinoma samples; $30.3 \%$ of the tumor samples showed medium expression and $52.5 \%$ showed high expression (Fig. 1A and 1B).

To assess the function of CLIP-170 in breast cancer, we inhibited its expression by using two different small interfering RNAs (siRNAs) and investigated cell proliferation by sulforhodamine B staining assay. Of the two CLIP-170 siRNAs, 
\#1 was designed to target both human and mouse CLIP-170 sequences, whereas \#2 was designed to target human CLIP170 only. The two siRNAs could efficiently decrease CLIP-170 expression in MCF7 and MDA-MB-231 human breast cancer cells (Fig. 1C and S1). Interestingly, knockdown of CLIP-170 expression did not significantly affect the proliferation of MCF7 cells and only slightly inhibited the proliferation of MDA-MB-231 cells (Fig. 1D and 1E). These data indicate that CLIP-170 is not critically involved in the proliferation of breast cancer cells, despite its elevated expression in breast tumor samples.

\section{CLIP-170 expression correlates positively with blood vessel density}

We next hypothesized that CLIP-170 is involved in breast cancer pathogenesis by stimulating tumor angiogenesis. To check this possibility, we examined its expression in the vasculature of human breast carcinoma by immunostaining with antibodies against CLIP-170 and the endothelial marker CD31. As shown in Fig. 2A, a significant amount of CLIP-170 was localized in the blood vessels. Importantly, there was a significant positive correlation between CLIP-170 expression and blood vessel density in breast carcinoma samples (Fig. 2B).

Depletion of CLIP-170 significantly inhibited tumor growth in mice by decreasing tumor vascularization

To further investigate the role of CLIP-170 in breast cancer, we studied the effects of CLIP-170 siRNAs on tumor vasculature and growth in mice. We first checked the efficiency of the siRNAs in inhibiting mouse CLIP-170 expression. Of the two CLIP-170 siRNAs, only \#1 could reduce CLIP-170 expression efficiently in NIH-3T3 mouse fibroblasts, and \#2 had no obvious effect, although both of the CLIP-170 siRNAs could inhibit CLIP-170 expression in MDA-MB-231 cells (Fig. 3A, 3B and S1). This result was consistent with the design of the CLIP-170 siRNAs; wherein \#1 siRNA was designed to target both human and mouse CLIP-170, \#2 siRNA was designed to target human CLIP-170 only.

We then examined the effects of CLIP-170 siRNAs on breast tumor growth in vivo by injecting subcutaneously MDAMB-231 cells and siRNAs in matrigel into athymic nude mice and measured tumor volume with a vernier caliper. We found that the tumor volume in the \#1 CLIP-170 siRNA group was much smaller than that in the control group; however, the tumor volume in the \#2 CLIP-170 siRNA group was similar to that in the control group (Fig. $3 \mathrm{C}$ ). This result indicates that the effect of CLIP-170 on tumor growth is independent of MDAMB-231 cell proliferation.

To investigate whether \#1 CLIP-170 siRNA inhibited tumor growth in mice by affecting the growth of mouse blood vessels into the human breast tumor, we sacrificed the mice and isolated tumors 24 days post-injection. Blood vessels were clearly visible on the surface of the isolated breast tumors (Fig. 3D). By immunostaining frozen sections of the tumors with an antibody against the endothelial marker endomucin,
A

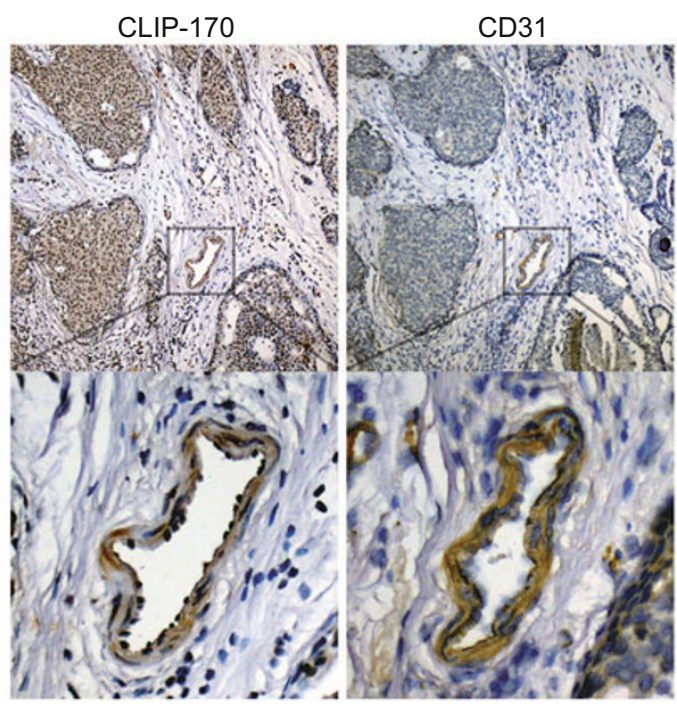

B

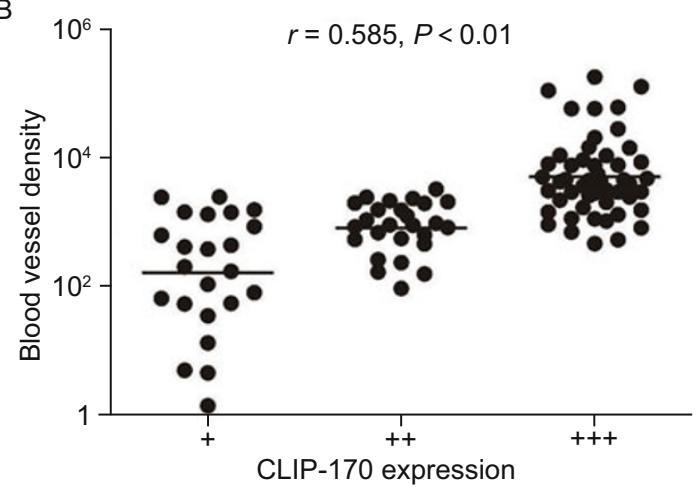

Figure 2. Expression of CLIP-170 in the vasculature of human breast carcinoma. (A) Immunohistochemistry of CLIP-170 and the endothelial marker CD31 in serial sections of breast carcinoma. (B) Correlation between CLIP-170 expression and blood vessel density in breast carcinoma analyzed by Spearman's rank correlation test.

we found that \#1, but not \#2, CLIP-170 siRNA significantly decreased tumor vascularization (Fig. 3E and 3F). Taken together, these data indicate that CLIP-170 promotes tumor growth in mice by mediating tumor angiogenesis.

\section{Knockdown of CLIP-170 expression impairs vascular endothelial tube formation and sprouting}

To substantiate the involvement of CLIP-170 in tumor angiogenesis, we examined CLIP-170 expression in human vascular endothelial cells in response to tumor cell induction. We found that conditioned media from MDA-MB-231 cells remarkably increased CLIP-170 expression in human umbilical vein endothelial cells (HUVECs) (Fig. 4A). We then analyzed the effect of CLIP-170 siRNAs on vascular endothelial tube formation in vitro. The two CLIP-170 siRNAs inhibited CLIP170 expression effectively in HUVECs (Fig. 4B and S1). We 
A

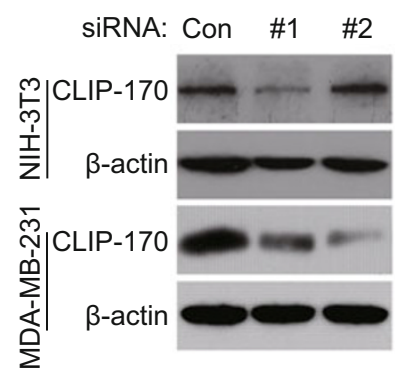

D

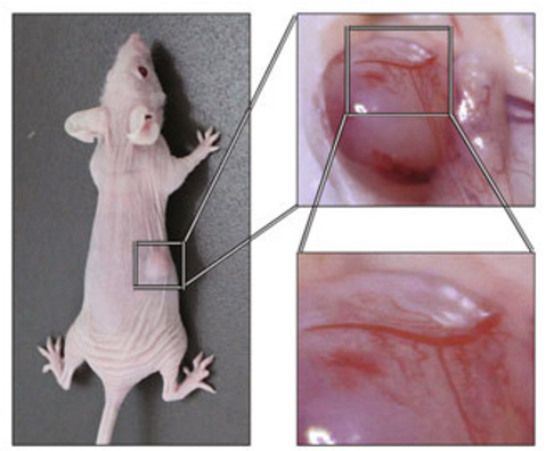

$\mathrm{F}$

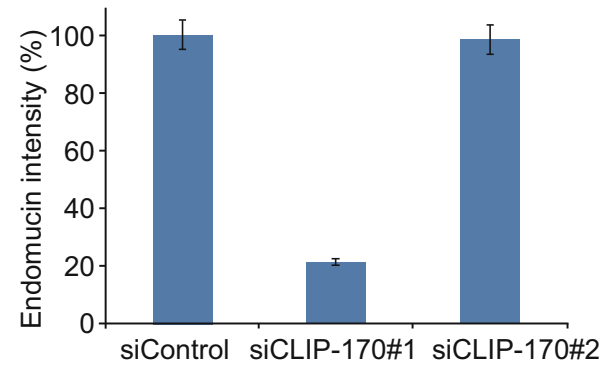

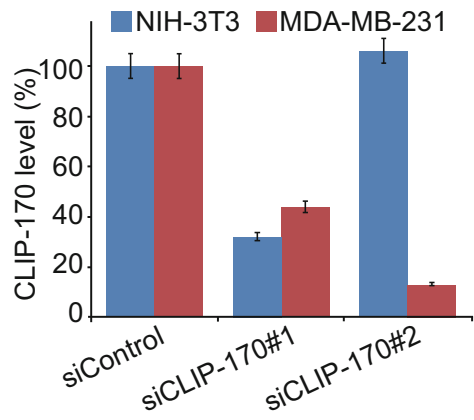

E
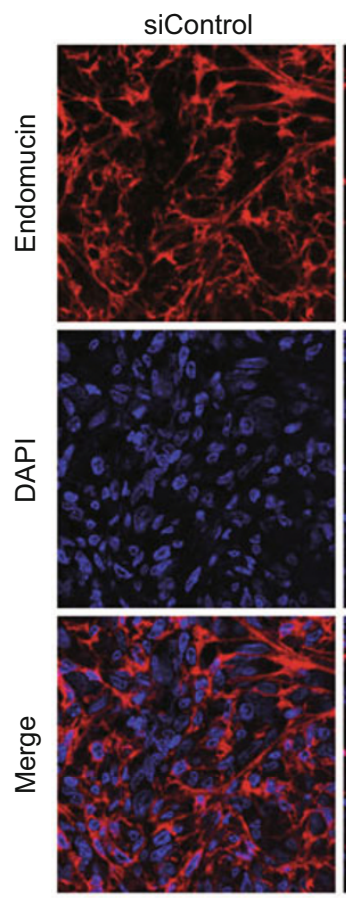

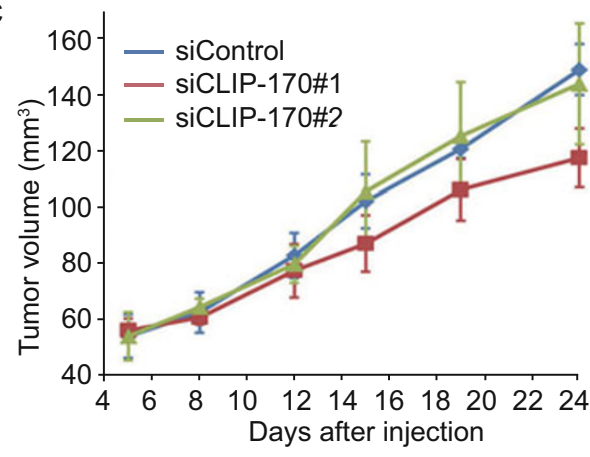

siCLIP-170\#1
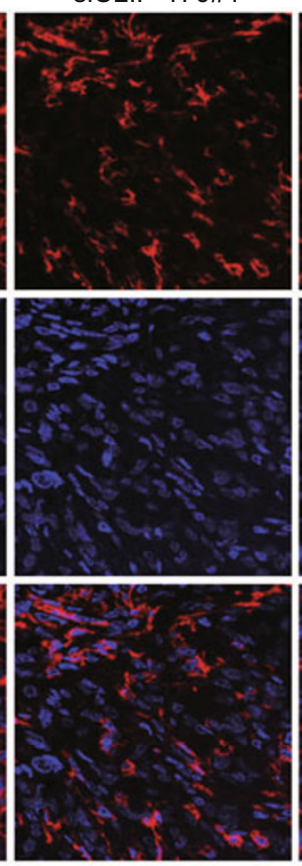

siCLIP-170\#2
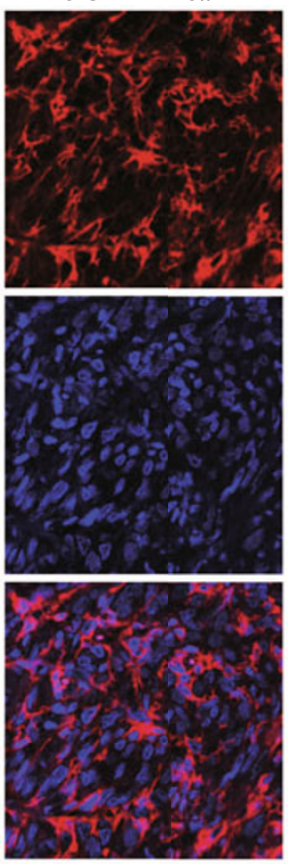

Figure 3. Effects of CLIP-170 siRNAs on tumor vasculature and growth in mice. (A) Western blot analysis of CLIP-170 and $\beta$-actin expression in NIH-3T3 mouse fibroblasts and MDA-MB-231 cells transfected with CLIP-170 or control siRNAs for $72 \mathrm{~h}$. (B) Experiments were performed as in A, and CLIP-170 expression level was determined by dividing the intensity of CLIP-170 with the intensity of $\beta$-actin on the blot. The data obtained were then normalized to the control group. (C) MDA-MB-231 cells and the indicated siRNAs in matrigel were injected subcutaneously into nude mice and the tumor volume was measured. (D) Representative image of the vasculature of human breast tumors grown in mice. (E) Frozen sections of breast tumors isolated from mice were stained with the endomucin antibody and DAPI and examined with a fluorescence microscope. $(F)$ Experiments were performed as in $E$, and endomucin intensity was quantified and normalized to the control group.

plated the siRNA-transfected HUVECs onto matrigel, added conditioned media from MDA-MB-231 cells, and then examined the formation of capillary/tube-like structures. As shown in Fig. 4C, CLIP-170 siRNAs remarkably impaired tube formation from HUVECs compared with the control group. By measuring the cumulative tube length, we found that the two CLIP-170 siRNAs inhibited tube formation by $62.9 \%$ and $65.0 \%$, respectively $3 \mathrm{~h}$ after plating cells onto matrigel (Fig. 4D). In addition, we found that overexpression of CLIP-170 in HUVECs significantly increased tube formation (Fig. 4E-G).
To further study the function of CLIP-170 in angiogenesis, we examined the effect of CLIP-170 siRNAs on endothelial sprouting in a collagen-based three-dimensional angiogenesis assay. We embedded the spheroids generated from HUVECs in collagen, added conditioned media from MDA-MB-231 cells on top of the gel, and then examined capillary-like endothelial sprouting. We found that CLIP-170 siRNAs significantly blocked endothelial sprouting from the spheroids compared with the control group (Fig. $4 \mathrm{H}$ and $4 \mathrm{I}$ ). Taken together, these results reveal that CLIP-170 is critically required for endothelial 
A

HUVEC

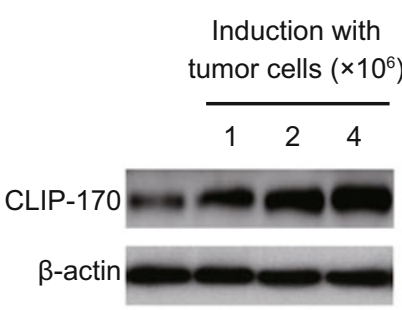

D
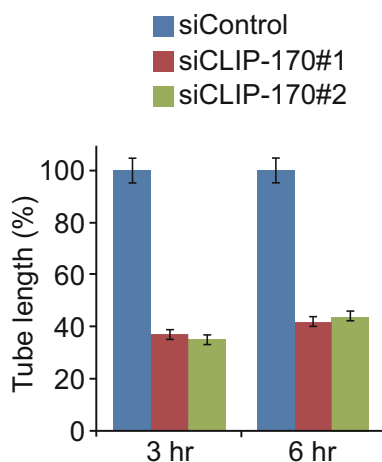

G

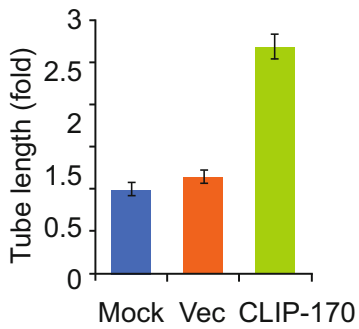

B

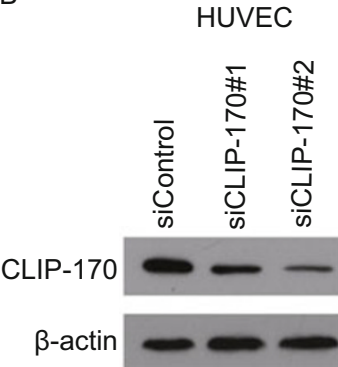

E

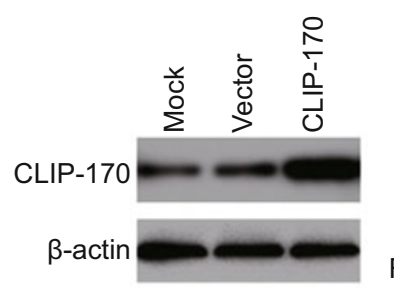

$\mathrm{F}$

siControl

siCLIP-170\#1

siCLIP-170\#2

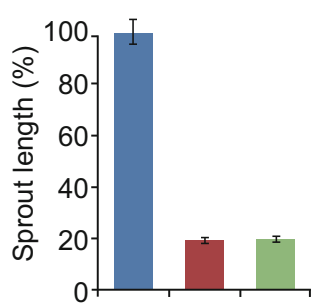

$\mathrm{H}$

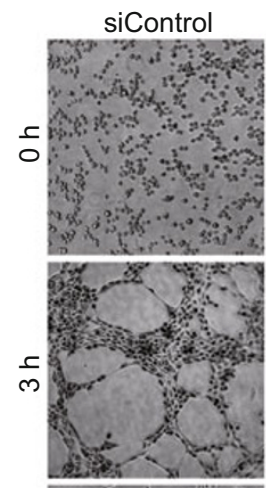

siCLIP-170\#1

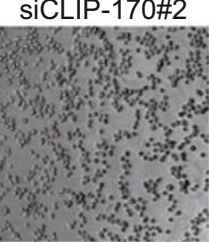

$\frac{5}{6}$
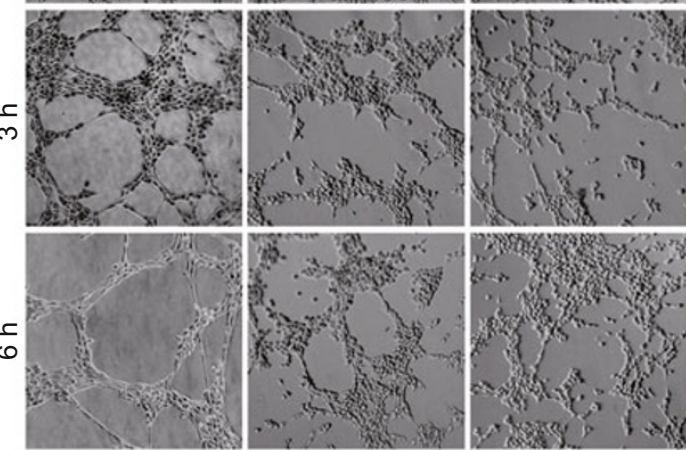

Mock
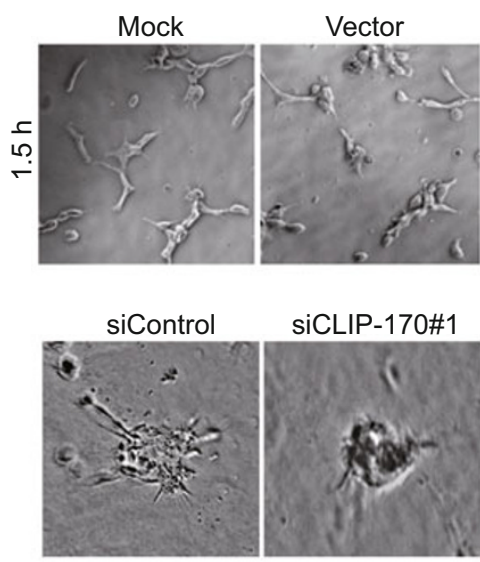

siCLIP-170\#1

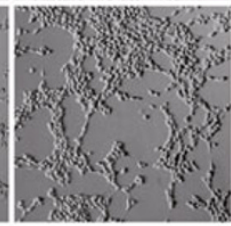

CLIP-170

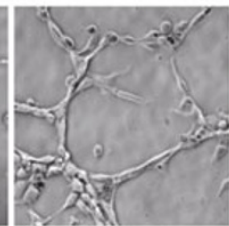

siCLIP-170\#2
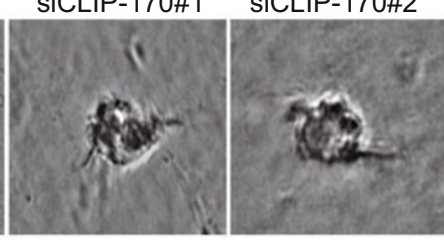

Figure 4. Knockdown of CLIP-170 expression impairs vascular endothelial tube formation and sprouting. (A) Western blot analysis of CLIP-170 and $\beta$-actin expression in HUVECs treated with conditioned media from $1 \times 10^{6}, 2 \times 10^{6}$, or $4 \times 10^{6}$ MDA-MB-231 cells for $24 \mathrm{~h}$. (B) Western blot analysis of CLIP-170 and $\beta$-actin expression in HUVECs transfected with CLIP-170 or control siRNAs for $72 \mathrm{~h}$. (C) HUVECs transfected with CLIP-170 or control siRNAs were plated onto matrigel, conditioned media from MDA-MB-231 cells were then added, and photographs were taken 0,3 , and $6 \mathrm{~h}$ later. (D) Experiments were performed as in (C), and the cumulative tube length was measured and normalized to the control group. (E) Western blot analysis of CLIP-170 and $\beta$-actin expression in HUVECs transfected pcDNA3-CLIP-170 or empty vector or untransfected (mock) for $24 \mathrm{~h}$. (F) HUVECs transfected with pcDNA3-CLIP-170 or empty vector or untransfected were plated onto matrigel, conditioned media from MDA-MB-231 cells were then added, and photographs were taken $1.5 \mathrm{~h}$ later. $(\mathrm{G})$ Experiments were performed as in $(\mathrm{F})$, and the cumulative sprout length was measured and normalized to the mock group. $(\mathrm{H})$ Capillary-like sprout formation from spheroids generated from HUVECs transfected with CLIP-170 or control siRNAs and cultured in conditioned media from MDA-MB-231 cells. (I) Experiments were performed as in $(\mathrm{H})$, and the cumulative sprout length was measured and normalized to the control group.

tube formation and sprouting in vitro.

\section{CLIP-170 is important for the migration but not the proliferation of vascular endothelial cells}

We then sought to dissect the molecular mechanisms underlying the function of CLIP-170 in angiogenesis. We first analyzed the effect of CLIP-170 siRNAs on the migration of vascular en- dothelial cells, which is a key step in the angiogenic process. We scratched the siRNA-transfected HUVECs to create the wound, added conditioned media from MDA-MB-231 cells, and then examined wound margins. By these in vitro wound healing experiments, we found that knockdown of CLIP-170 expression dramatically delayed wound closure, indicating compromised cell migration (Fig. 5A and 5B). In addition, transwell migration assays showed that CLIP-170 siRNAs remark- 
A

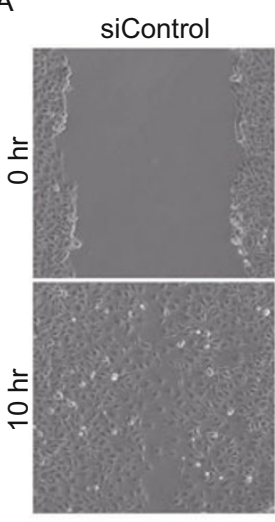

E

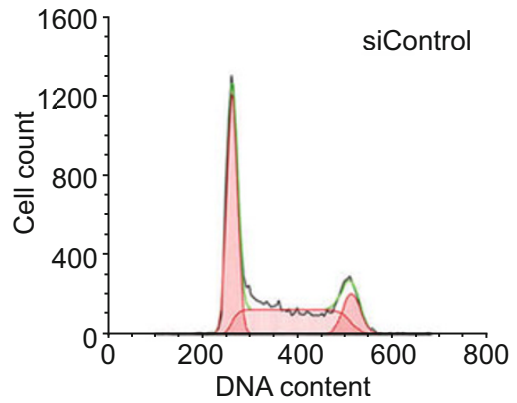

$\mathrm{F}$

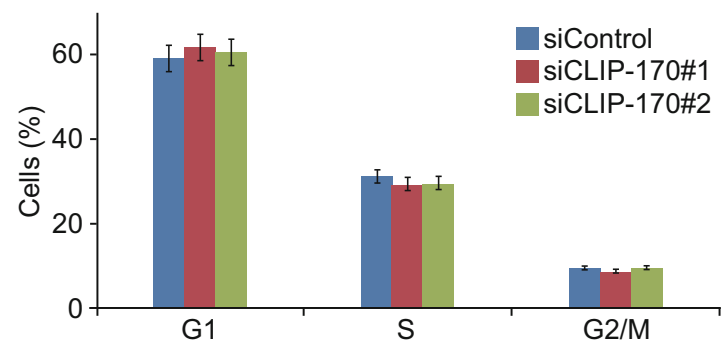

B
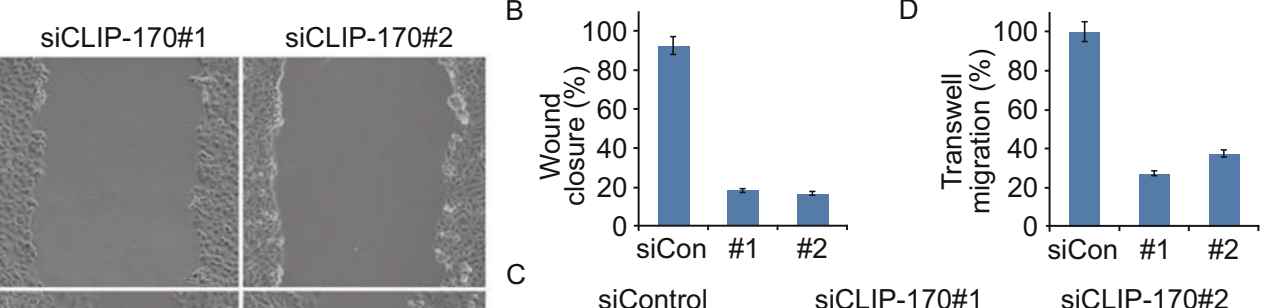

C

$$
\text { siControl siCLIP-170\#1 }
$$
siCLIP-170\#2
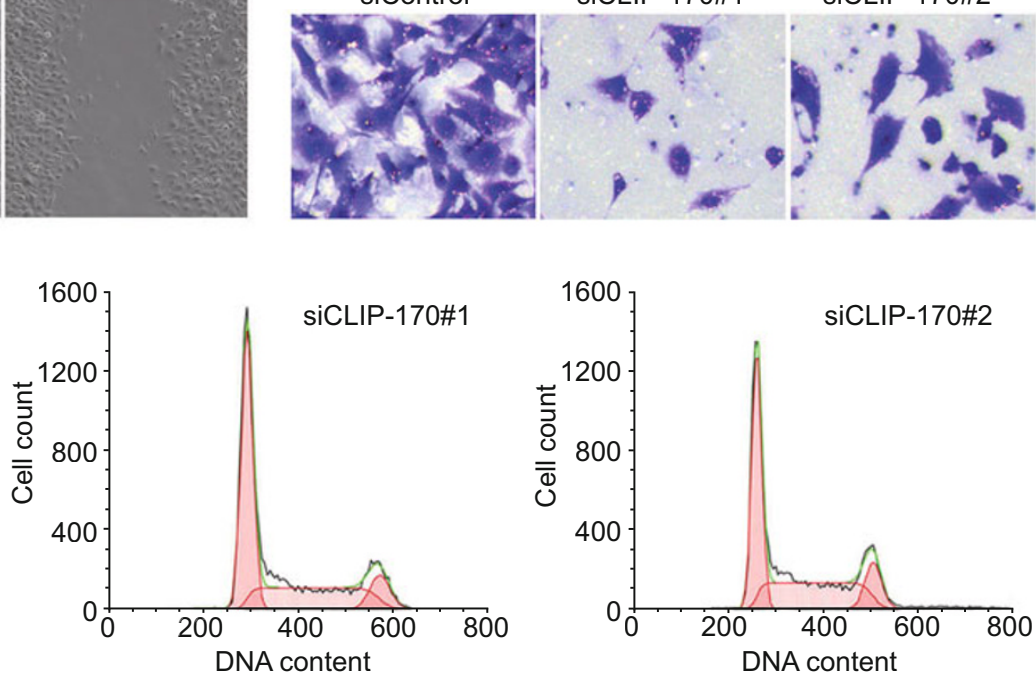

G

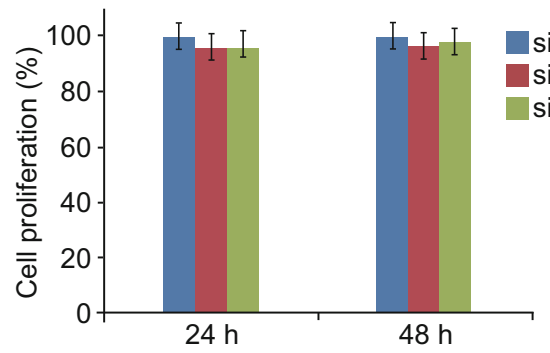

Figure 5. Effects of CLIP-170 siRNAs on endothelial cell migration and proliferation. (A) HUVECs transfected with CLIP-170 or control siRNAs for $72 \mathrm{~h}$ were scratched, conditioned media from MDA-MB-231 cells were then added, and wound margins were imaged 0 and $10 \mathrm{~h}$ later. (B) Experiments were performed as in (A), and the extent of wound closure was quantified by measuring the wound area compared with the initial wound area. (C) HUVECs transfected with CLIP-170 or control siRNAs were plated onto the inside of the transwell insert, and the insert was placed in a 24-well plate containing conditioned media from MDA-MB-231 cells. After $18 \mathrm{~h}$, cells migrated to the underside of the insert were stained with crystal violet. (D) Experiments were performed as in (C), and the number of migrated cells was examined and normalized to the control group. (E) Flow cytometric analysis of DNA content of HUVECs transfected with CLIP-170 or control siRNAs. (F) Experiments were performed as in (E), and the percentages of cells in $G 1, S$, and $G 2 / M$ phases were quantified. (G) HUVECs were transfected with CLIP-170 or control siRNAs and cell proliferation was examined by sulforhodamine B staining.

ably inhibited the migration of HUVECs through the porous membrane (Fig. 5C and 5D).

Given that both the migration and proliferation of vascular endothelial cells are important for angiogenesis (Folkman, 2007; Carmeliet and Jain, 2011; Weis and Cheresh, 2011), it is possible that CLIP-170 stimulates angiogenesis via a role in cell proliferation, in addition to its role in cell migration. To test this possibility, we analyzed the effects of CLIP-170 siRNAs on the cell cycle and proliferative rate of HUVECs. By flow cytometric analysis of DNA content, we found that knockdown of CLIP-170 expression did not obviously affect the cell cycle of endothelial cells (Fig. 5E and 5F). In addition, sulphorhodamine B staining assay revealed that CLIP-170 siRNAs did not significantly affect endothelial cell proliferation (Fig. 5G). These data therefore suggest that CLIP-170 regulates angiogenesis by mediating the migration but not the proliferation of endothelial cells.

\section{CLIP-170 is critical for endothelial cell polarization}

To gain more mechanistic insight into the role of CLIP-170 in 

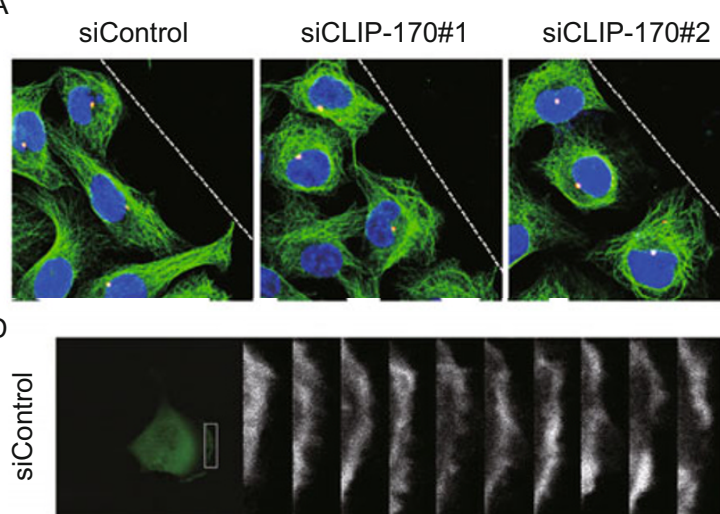

莫
$\frac{1}{5}$
$\frac{0}{\infty}$

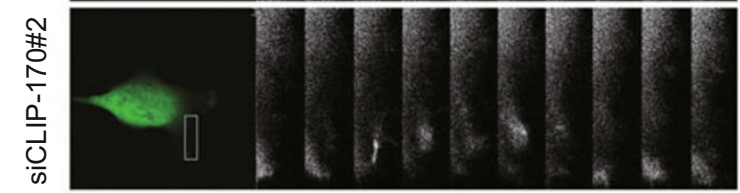

B

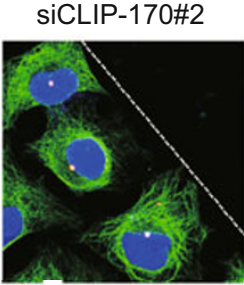

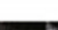

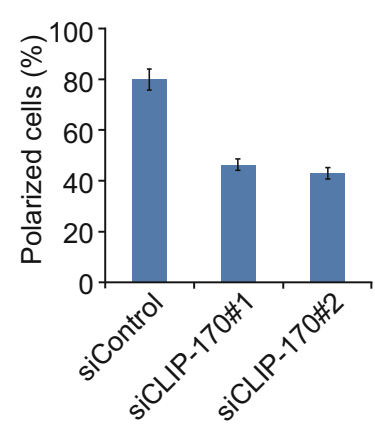

E

siControl

siCLIP-170\#1

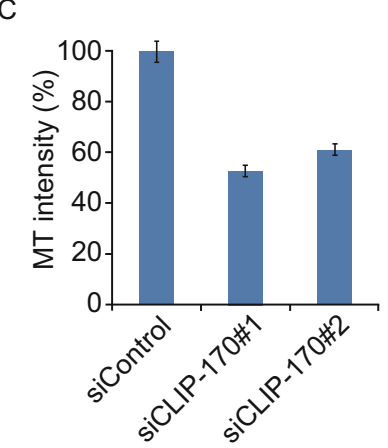

255

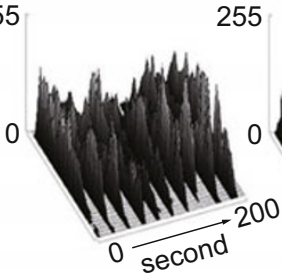

255

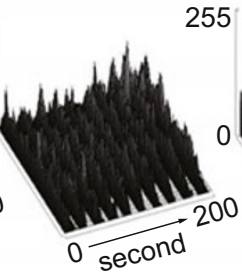

siCLIP-170\#2

Figure 6. CLIP-170 is critical for endothelial cell polarization. (A) HUVECs transfected with CLIP-170 or control siRNAs were scratched, conditioned media from MDA-MB-231 cells were added, and cells were fixed $2 \mathrm{~h}$ later and stained with anti- $\alpha$-tubulin antibody, anti-pericentrin antibody, and DAPI to visualize microtubules (green), centrosomes (red), and nuclei (blue), respectively. Broken white lines indicate the wound direction. (B) Experiments were performed as in A, and the percentage of polarized cells at the wound margin was quantified. (C) Experiments were performed as in A, and the intensity of leading-edge microtubules at the wound margin was quantified and normalized to the control group. (D) HUVECs were transfected with the pEGFPC1 plasmid and the indicated siRNAs, and the fluorescence of GFP at the leading edge of cells was recorded. Rectangular regions in the leading edge were randomly selected to analyze membrane ruffle dynamics. (E) Experiments were performed as in $D$, and membrane ruffle dynamics were presented as threedimensional surface plots.

angiogenesis, we investigated its potential involvement in the polarization of vascular endothelial cells, which is critical for their directional migration toward tumor cells (Vicente-Manzanares and Horwitz, 2011). We scratched the siRNA-transfected HUVECs to create the wound, added conditioned media from MDA-MB-231 cells, and examined cell morphology $2 \mathrm{~h}$ later. By immunofluorescence microscopy, we found that cells at the wound margin had a typical polarized structure, with the centrosome positioned between the nucleus and the cell front within a $120^{\circ}$-angle facing the wound (Fig. 6A). In contrast, the polarized morphology was impaired in cells transfected with CLIP-170 siRNAs (Fig. 6A). By quantifying the percentage of polarized cells at the wound margin and the intensity of leading-edge microtubules at the wound margin, we found that knockdown of CLIP-170 expression significantly inhibited cell polarization (Fig. 6B and 6C).

To corroborate the involvement of CLIP-170 in cell polarization and migration, membrane ruffle dynamics at the leading edge of migrating cells were assessed via live cell imaging. We transfected HUVECs with control or CLIP-170 siRNAs together with a plasmid expressing GFP, scratched the cells, and then recorded GFP fluorescence at the leading edge of transfected cells (Fig. 6D). The sequential fluorescence images were then transformed to three-dimensional surface plots indicating dynamic changes in GFP fluorescence. As shown in Fig. 6E, inhibition of CLIP-170 expression remarkably suppressed the dynamic changes in GFP fluorescence at the leading edge of migrating cells, suggesting diminished membrane ruffle dynamics. These results thus provide further evidence that CLIP-170 regulates the polarization and migration of vascular endothelial cells.

\section{DISCUSSION}

Tumor-associated angiogenesis involves a complex interplay of a variety of cell types in the tumor microenvironment. This striking feature raises therapeutic opportunities for targeting multiple aspects of angiogenesis to inhibit tumor progression (Folkman, 2007; Carmeliet and Jain, 2011; Weis and Cheresh, 2011). Current antiangiogenic strategies, primarily those targeting vascular endothelial growth factor and its receptors, are showing promise in several cancer types including breast cancer (Bossung and Harbeck, 2010; Mackey et al., 2012). However, to improve the therapeutic response, it is crucial 


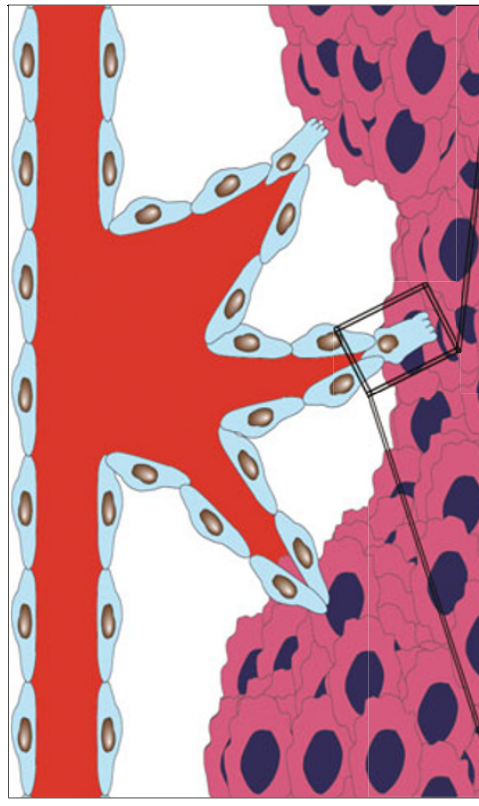

Tumor angiogenesis

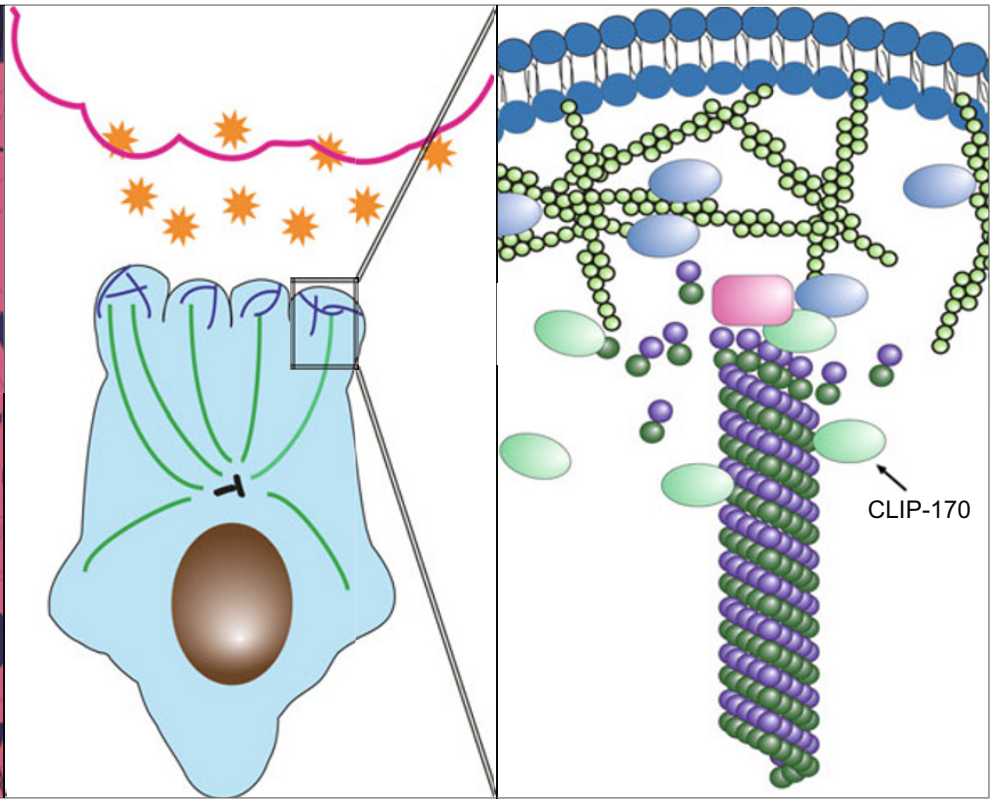

Cell polarization and migration

Microtubule stabilization

Figure 7. A model illustrating the role of CLIP-170 in tumor angiogenesis. In response to angiogenic signals released from tumor cells, CLIP-170 promotes the cortical capture and stabilization of microtubules in vascular endothelial cells and therefore stimulates their polarization and migration, leading to blood vessel growth toward tumor cells.

to identify additional antiangiogenic targets, especially those highly expressed or activated in cancer specimens. In the present study, we have identified the microtubule-binding protein CLIP-170 as a critical regulator of angiogenesis in breast carcinoma by the following evidence: a) this protein is highly expressed in the majority of breast carcinoma samples; b) its expression correlates positively with blood vessel density in the tumor samples; c) siRNA-mediated reduction of CLIP-170 expression dramatically inhibited vascular endothelial tube formation and sprouting in vitro; and d) depletion of its expression suppressed breast tumor growth in mice by decreasing tumor vascularization. Thus CLIP-170 joins a growing list of proteins regulating tumor angiogenesis and possesses the potential as a novel antiangiogenic target.

Several previous studies have implicated CLIP-170 in the pathogenesis of cancer. For example, overexpression of a splicing variant of CLIP-170 has been observed in Hodgkin's disease and anaplastic large-cell lymphoma (Bilbe et al., 1992; Delabie et al., 1992). CLIP-170 has also been shown to regulate lamellipodium formation and invasion of breast cancer cells (Suzuki and Takahashi, 2008). In addition, CLIP-170 expression has recently been reported to influence the pathological response of breast cancer patients to chemotherapy (Sun et al., 2012). However, the molecular mechanisms of how CLIP-170 is involved in tumor development are not fully understood. Our data demonstrate that CLIP-170 stimulates tumor angiogenesis by acting on endothelial cells and is not critically involved in the proliferation of tumor cells. These findings thus underscore the importance of the tumor-endothelial cell interaction in the tumor microenvironment and reveal a critical function of CLIP-170 in the angiogenic response. It should be noted, however, that CLIP-170 is involved in a wide spectrum of cellular processes and therefore may participate in the pathogenesis of cancer through alternative mechanisms in addition to the proangiogenic role.

Our results also provide mechanistic insight into how CLIP170 promotes tumor angiogenesis. By in vitro wound healing and transwell assays, we show that CLIP-170 mediates the migration of vascular endothelial cells toward angiogenic signals. In addition, by measuring centrosome position, leading-edge microtubule intensity, and membrane ruffle dynamics, we demonstrate that CLIP-170 regulates the polarization and migration of vascular endothelial cells in response to the angiogenic stimulus. Our data also reveal that CLIP-170 is not critical for the cell cycle or proliferation of vascular endothelial cells. Collectively, these findings suggest a working model for the action of CLIP-170 in tumor angiogenesis. In this model, upon release of angiogenic signals from tumor cells, CLIP-170 in endothelial cells of nearby blood vessels promotes microtubule capture and stabilization by the cell cortex. Microtubule stabilization in turn contributes to neovasculature formation by promoting endothelial cell polarization and migration (Fig. 7). These results thus expand our understanding of tumor angiogenesis and link the classic function of CLIP-170 in microtubule-associated cellular processes to its pathological function in cancer.

It is worthy of note that CLIP-170 is critical for diverse normal physiological processes. Therefore, antiangiogenic strategies targeting CLIP-170 to inhibit tumor progression might have 
side effects. However, given that CLIP-170 is highly expressed and correlates positively with vascular density in breast tumor and that targeting endothelial cells offers therapeutic benefit (Bossung and Harbeck, 2010; Mackey et al., 2012), inhibiting CLIP-170 alone or in combination with other therapeutics might be useful for the management of breast cancer. In the present study, by injecting subcutaneously breast cancer cells and CLIP-170 siRNAs in matrigel into mice, we found that CLIP170 siRNAs could inhibit breast tumor growth in vivo by reducing tumor vascularization. To further evaluate the therapeutic efficacy of CLIP-170 siRNAs in vivo, it would be important to systemically deliver CLIP-170 siRNAs into the tumor-associated vasculature using an orthotopic model of breast carcinoma and then examine the effects of CLIP-170 gene silencing on tumor growth.

\section{MATERIALS AND METHODS}

Chemicals, antibodies, plasmids, and siRNAs

Propidium iodide, 4',6-diamidino-2-phenylindole (DAPI), and sulforhodamine B were purchased from Sigma-Aldrich (St. Louis, MO). Matrigel and collagen were obtained from BD Biosciences (San Jose, CA). Antibodies against CLIP-170, pericentrin, endomucin, and Ki-67 (Santa Cruz Biotechnology, Santa Cruz, CA), $\beta$-actin (Sigma-Aldrich), and CD31 (BioLegend, San Diego, CA) were obtained from the indicated sources. Horseradish peroxidase-conjugated secondary antibodies were from Amersham Biosciences (Chandler, AZ), and fluoresceinor rhodamine-conjugated secondary antibodies were from Jackson ImmunoResearch Laboratories (West Grove, PA). The pEGFPC1 plasmid was from Clontech (Mountain View, CA), and the pcDNA3CLIP-170 plasmid was constructed by insertion of the CLIP-170 CDNA into the pcDNA3 vector. CLIP-170 siRNAs (\#1: 5'-UGAAGAUGUCAGGAGAUAA-3'; \#2: 5'-UUUAGCUGCUUCUUGCUGGCUCUGU-3') and luciferase control siRNA (5'-CGUACGCGGAAUACUUCGA-3') were synthesized by Invitrogen (Carlsbad, CA).

\section{Cell culture and transfection}

MCF7 and MDA-MB-231 breast cancer cells and NIH-3T3 mouse fibroblasts were obtained from the American Type Culture Collection (ATCC, Manassas, VA) and cultured in RPMI 1640 medium supplemented with $10 \%$ fetal bovine serum at $37^{\circ} \mathrm{C}$ in a humidified atmosphere with $5 \% \mathrm{CO}_{2}$. HUVECs were also from the ATCC and maintained as described previously (Gao et al., 2010). Transfection of plasmids and siRNAs was performed by using the Lipofectamine 2000 reagent (Invitrogen)

\section{Immunohistochemistry}

Human breast tissues were obtained from 98 patients undergoing surgical resection in 2008 and 2009 at Shanxian Dongda Hospital and immunostained as described (Wang et al., 2009; Dong et al., 2010). In brief, paraffin-embedded breast tissue sections were cut, dewaxed, and rehydrated with xylene and graded alcohols. Antigen retrieval was carried out in $5 \mathrm{mmol} / \mathrm{L}$ citrate buffer. After inactivation of endogenous peroxidase with $3 \% \mathrm{H}_{2} \mathrm{O}_{2}$, the sections were blocked with goat serum and incubated with primary antibodies. The sections were then incu- bated with biotinylated secondary antibody and streptavidin-biotinperoxidase, using 3,3'-diaminobenzidine as a chromogen. Finally, the sections were counterstained with haematoxylin. CLIP-170 expression level was graded based on the intensity of staining $(0=$ negative; $1=$ low; $2=$ medium; $3=$ high $)$ and the percentage of stained cells ( $0=0 \%$ stained; $1=1 \%-25 \%$ stained; $2=26 \%-50 \%$ stained; $3=51 \%-100 \%$ stained) as described previously (Wang et al., 2009; Dong et al., 2010). A multiplied score (intensity score $\times$ percentage score) $<2$ was considered as negative staining (-), 2-3 as low staining (+), 4-6 as medium staining $(++)$ and $>6$ as high staining $(+++)$. The percentage of tissue samples with different levels of CLIP-170 expression among all the tissue samples was then calculated.

Measurement of the correlation between blood vessel density and CLIP-170 expression

Serial sections of breast carcinoma were immunostained with antibodies against CLIP-170 and the endothelial marker CD31, and CLIP-170 expression level was graded as described above. Tumor blood vessel density was determined by measuring the intensity of CD31 in an entire section and then dividing by the total area of tumor present in the section. The correlation between blood vessel density and CLIP-170 expression was then analyzed by Spearman's rank correlation test to obtain the correlation coefficient $(r)$ and $P$ value.

\section{Western blot analysis}

Cells were lysed in a buffer containing 1\% Triton X-100, $150 \mathrm{mmol} / \mathrm{L}$ $\mathrm{NaCl}$, and $50 \mathrm{mmol} / \mathrm{L}$ Tris (pH 7.5). Proteins were resolved by sodium dodecyl sulfate-polyacrylamide gel electrophoresis and transferred onto polyvinylidene difluoride membranes (Millipore, Billerica, MA). The membranes were blocked in Tris-buffered saline containing $0.2 \%$ Tween 20 and $5 \%$ fat-free dry milk and incubated first with primary antibodies and then with horseradish peroxidase-conjugated secondary antibodies. Specific proteins were visualized with enhanced chemiluminescence detection reagent according to the manufacturer's instructions (Pierce Biotechnology, Rockford, IL).

Immunofluorescence microscopy

Cells grown on glass coverslips were fixed with $4 \%$ paraformaldehyde for $30 \mathrm{~min}$ at room temperature. Cells were blocked with $2 \%$ bovine serum albumin in phosphate-buffered saline, incubated with primary antibodies, and then with fluorescein- and/or rhodamine-conjugated secondary antibodies followed by staining with DAPI for 5 min as described previously (Sun et al., 2008). Coverslips were mounted with $90 \%$ glycerol in phosphate-buffered saline and examined with the Axio Observer A1 fluorescence microscope (Carl Zeiss, Oberkochen, Germany).

\section{Live cell imaging}

HUVECs were cultured in a $37^{\circ} \mathrm{C}$ chamber on a TCS SP5 confocal microscope (Leica, Wetzlar, Germany) equipped with a live-cell imaging workstation. The fluorescence of GFP at the leading edge of cells was recorded at 20-s intervals with the use of the LASAF software (Leica). The acquired image sequences were analyzed by ImageJ (National Institutes of Health, Bethesda, MD), and membrane ruffle dynamics were presented as three-dimensional surface plots. 


\section{Animal experiments}

MDA-MB-231 cells $\left(5 \times 10^{5}\right)$ and siRNAs in matrigel were injected subcutaneously into the right flanks of female athymic nude mice (six mice per group) as described (Chae et al., 2004; Liu et al., 2010). Tumor volume was measured with a vernier caliper and calculated with the following formula: $V=\pi / 6 \times$ length $\times$ width $^{2}$. The mice were sacrificed 24 days post-injection, and tumors were isolated for the measurement of Ki-67 or endomucin intensity.

\section{Endothelial tube formation and sprouting assays}

To examine tube formation, HUVECs were plated onto $35-\mathrm{mm}$ culture dishes precoated with matrigel, and conditioned media from MDAMB-231 cells were then added. Photographs were taken at different time points, and the cumulative tube length was measured with the Image J software. The data obtained were then normalized to the control group. To examine capillary sprouting, cell spheroids were generated by culturing HUVECs overnight in medium containing $0.25 \%$ carboxymethylcellulose in round-bottom 96-well plates. The spheroids were embedded into collagen and transferred to 24-well plates. Conditioned media from MDA-MB-231 cells were then added on top of the gel. Capillary-like sprout formation was examined by microscopy, and the cumulative sprout length per spheroid was measured with the ImageJ software. The data obtained were then normalized to the control group.

\section{Cell migration assays}

To analyze cell migration by wound healing, confluent monolayers of serum-starved cells cultured in 24-well plates were scratched with a $10-\mathrm{mL}$ pipette tip to generate the wound. Cells were washed with phosphate-buffered saline, and conditioned media from MDA-MB-231 cells were added. Phase-contrast photographs of the wound were taken at different time points. The extent of wound closure was determined by measuring with the Image J software the area of cells that migrated into the wound and then dividing by the total area of wound. Transwell cell migration assays were performed as described previously (Shi et al., 2012). Briefly, HUVECs suspended in serum-free medium were added to the inside of the transwell insert precoated with matrigel, and the insert was then placed in a 24-well plate containing conditioned media from MDA-MB-231 cells. After $18 \mathrm{~h}$, cells on the inside of the transwell insert were removed with a cotton swab, and cells on the underside of the insert were fixed with $4 \%$ paraformaldehyde and stained with crystal violet solution. Three randomly selected fields were photographed, and the number of migrated cells was counted. The data obtained were then normalized to the control group.

\section{Measurement of cell polarization}

Serum-starved cells grown as confluent monolayers were scratched, and conditioned media from MDA-MB-231 cells were added. Cells were fixed $2 \mathrm{~h}$ later and immunostained for microtubules, centrosomes, and nuclei. The percentage of polarized cells at the wound margin was then quantified by counting; polarized cells were referred to cells with the centrosome positioned between the nucleus and the cell front, within a $120^{\circ}$-angle facing the wound. The intensity of leading-edge microtubules at the wound margin was quantified with the Image J software; leading-edge microtubules were referred to cellular microtubules within a $120^{\circ}$-angle facing the wound. The data obtained were then normalized to the control group.

\section{Cell proliferation assay}

Cells were seeded at $5 \times 10^{4}$ cells per well in 24-well tissue culture plates. After various days, cells were fixed with $50 \%$ trichloroacetic acid and stained with $0.4 \%$ sulforhodamine $B$ dissolved in $1 \%$ acetic acid. The cells were then washed with $1 \%$ acetic acid to remove unbound dye. The protein-bound dye was extracted with $10 \mathrm{mmol} / \mathrm{L}$ Tris base to determine the optical density at $490 \mathrm{~nm}$ wavelength.

Ethical declaration

The use of human samples in this study was approved by the Ethics Committee of Nankai University, and written informed consent was received from participants prior to inclusion in the study. The use of mice was approved by the Animal Care and Use Committee of Nankai University.

\section{ACKNOWLEDGEMENTS}

We are grateful to Shanxian Dongda Hospital for kindly providing breast cancer samples, Xinglong Zhou, Ruming Liu, and Qiang Zhao for experimental assistance, and members of the Zhou laboratory for discussion. This work was supported by grants from the National Basic Research Program (973 Program) (Nos. 2012CB945002, 2010CB912204), National Natural Science Foundation of China (Grant Nos. 31130015 and 31271437), and the 111 project from the Ministry of Education of China (B08011).

\section{REFERENCES}

Akhmanova, A., and Steinmetz, M.O. (2008). Tracking the ends: a dynamic protein network controls the fate of microtubule tips. Nat Rev Mol Cell Biol 9, 309-322.

Bilbe, G., Delabie, J., Bruggen, J., Richener, H., Asselbergs, F.A., Cerletti, N., Sorg, C., Odink, K., Tarcsay, L., Wiesendanger, W., et al. (1992). Restin: a novel intermediate filament-associated protein highly expressed in the Reed-Sternberg cells of Hodgkin's disease. EMBO J 11, 2103-2113.

Bornens, M. (2008). Organelle positioning and cell polarity. Nat Rev Mol Cell Biol 9, 874-886.

Bossung, V., and Harbeck, N. (2010). Angiogenesis inhibitors in the management of breast cancer. Curr Opin Obstet Gynecol 22, 79-86.

Carmeliet, P., and Jain, R.K. (2011). Molecular mechanisms and clinical applications of angiogenesis. Nature 473, 298-307.

Chae, S.S., Paik, J.H., Furneaux, H., and Hla, T. (2004). Requirement for sphingosine 1-phosphate receptor-1 in tumor angiogenesis demonstrated by in vivo RNA interference. J Clin Invest 114, 1082-1089.

Delabie, J., Shipman, R., Bruggen, J., De Strooper, B., van Leuven, F., Tarcsay, L., Cerletti, N., Odink, K., Diehl, V., Bilbe, G., et al. (1992). Expression of the novel intermediate filament-associated protein restin in Hodgkin's disease and anaplastic large-cell lymphoma. Blood 80, 2891-2896.

Dong, X., Liu, F., Sun, L., Liu, M., Li, D., Su, D., Zhu, Z., Dong, J.T., Fu, L., and Zhou, J. (2010). Oncogenic function of microtubule endbinding protein 1 in breast cancer. J Pathol 220, 361-369.

Folkman, J. (2007). Angiogenesis: an organizing principle for drug dis- 
covery? Nat Rev Drug Discov 6, 273-286.

Gao, J., Sun, L., Huo, L., Liu, M., Li, D., and Zhou, J. (2010). CYLD regulates angiogenesis by mediating vascular endothelial cell migration. Blood 115, 4130-4137.

Gouveia, S.M., and Akhmanova, A. (2010). Cell and molecular biology of microtubule plus end tracking proteins: end binding proteins and their partners. Int Rev Cell Mol Biol 285, 1-74.

Lamalice, L., Le Boeuf, F., and Huot, J. (2007). Endothelial cell migration during angiogenesis. Circ Res 100, 782-794.

Li, D., Xie, S., Ren, Y., Huo, L., Gao, J., Cui, D., Liu, M., and Zhou, J. (2011). Microtubule-associated deacetylase HDAC6 promotes angiogenesis by regulating cell migration in an EB1-dependent manner. Protein Cell 2, 150-160.

Li, J., Sun, X., Wang, Z., Chen, L., Li, D., Zhou, J., and Liu, M. (2012). Regulation of vascular endothelial cell polarization and migration by Hsp70/Hsp90-organizing protein. PLoS One 7, e36389.

Li, R., and Gundersen, G.G. (2008). Beyond polymer polarity: how the cytoskeleton builds a polarized cell. Nat Rev Mol Cell Biol 9, 860-873.

Liu, M., Wang, X., Yang, Y., Li, D., Ren, H., Zhu, Q., Chen, Q., Han, S., Hao, J., and Zhou, J. (2010). Ectopic expression of the microtubuledependent motor protein Eg5 promotes pancreatic tumourigenesis. J Pathol 221, 221-228.

Mackey, J.R., Kerbel, R.S., Gelmon, K.A., McLeod, D.M., Chia, S.K., Rayson, D., Verma, S., Collins, L.L., Paterson, A.H., Robidoux, A., et al. (2012). Controlling angiogenesis in breast cancer: a systematic review of anti-angiogenic trials. Cancer Treat Rev 38, 673-688.

Peng, G., Ren, Y., Sun, X., Zhou, J., and Li, D. (2012). Inhibition of farnesyltransferase reduces angiogenesis by interrupting endothe- lial cell migration. Biochem Pharmacol 83, 1374-1382.

Rodriguez, O.C., Schaefer, A.W., Mandato, C.A., Forscher, P., Bement, W.M., and Waterman-Storer, C.M. (2003). Conserved microtubuleactin interactions in cell movement and morphogenesis. Nat Cell Biol 5, 599-609.

Shi, X., Liu, M., Li, D., Wang, J., Aneja, R., and Zhou, J. (2012). Cep70 contributes to angiogenesis by modulating microtubule rearrangement and stimulating cell polarization and migration. Cell Cycle 11, 1554-1563.

Sun, L., Gao, J., Dong, X., Liu, M., Li, D., Shi, X., Dong, J.T., Lu, X., Liu, C., and Zhou, J. (2008). EB1 promotes Aurora-B kinase activity through blocking its inactivation by protein phosphatase $2 A$. Proc Natl Acad Sci U S A 105, 7153-7158.

Sun, X., Li, D., Yang, Y., Ren, Y., Li, J., Wang, Z., Dong, B., Liu, M., and Zhou, J. (2012). Microtubule-binding protein CLIP-170 is a mediator of paclitaxel sensitivity. J Pathol 226, 666-673.

Suzuki, K., and Takahashi, K. (2008). Regulation of lamellipodia formation and cell invasion by CLIP-170 in invasive human breast cancer cells. Biochem Biophys Res Commun 368, 199-204.

Vicente-Manzanares, M., and Horwitz, A.R. (2011). Cell migration: an overview. Methods Mol Biol 769, 1-24.

Wang, H., Liu, B., Zhang, C., Peng, G., Liu, M., Li, D., Gu, F., Chen, Q., Dong, J.T., Fu, L., et al. (2009). Parkin regulates paclitaxel sensitivity in breast cancer via a microtubule-dependent mechanism. $\mathrm{J}$ Pathol 218, 76-85.

Watanabe, T., Noritake, J., and Kaibuchi, K. (2005). Regulation of microtubules in cell migration. Trends Cell Biol 15, 76-83.

Weis, S.M., and Cheresh, D.A. (2011). Tumor angiogenesis: molecular pathways and therapeutic targets. Nat Med 17, 1359-1370. 